\title{
Transport of Trichloroethylene Vapor in a Dry Soil Column
}

\author{
Chiu-Shia Fen, Po-Yang Yen \\ Department of Environmental Engineering and Science, Feng Chia University \\ Wenhwa Rd. No. 100, Taichung, Taiwan, ROC
}

\section{Extended Abstract}

Reliable prediction of the unsaturated zone transport of volatile organic compound (VOC) plumes in soil and groundwater is essential for adequately assessing their impact on the surrounding environment and resident. VOC plumes are usually leached from shallow source zones as such sources may persist for decades. Dense nonaqueous-phase liquids (DNAPLs) such as chlorinated solvent, e.g., Trichloroethylene (TCE), are frequently found in contaminated soils and groundwater. Their vapors migrating to ground surface cause environmental risks and pose a threat to human health. Understanding the mechanisms of VOC vapor transport at contaminated sites is important for reducing such risks. Fen and Abriola (2004) demonstrated range of errors that may be encountered in model applications to purely diffusive transport system due to use of models with different diffusive approaches. VOC vapor transport from source zones may be affected by gravitational force such that density-driven flow may also be significant (Falta et al., 1989). Fen et al. (2017) found a Dusty Gas Model (DGM)-based gas phase transport model and a molar-based Fickian-type diffusion model may produce substantially different dense gas density evolution profiles for vertically upward transport of a dense gas along a 40-cm soil column. The DGM predictions, however, may match the observations better than the Fickian results do. Thus, this study further explores vertically upward migration of TCE vapor in a small dry soil column at a length of $11 \mathrm{~cm}$. Transport experiments were conducted in a soil column with TCE liquid contained in a reservoir connected at one end of the column and a 5-cm head space at the other end. Time-varied pressure differences between the column ends and vapor concentrations at the column ends were measured for 8 to 50 hours. Two gas phase transport models, Michigan Soil Vapor Extraction Remediation (MISER) (Abriola et al., 1997) and Dusty Gas Model-based Gas Phase Transport (DGPT) (Fen, 2014), were applied to simulate the transport scenarios of the experiment in order to assess their predictability.

The results of three data sets measured from the transport experiment show that the upstream gas pressures (at the bottom of the column) are more than the downstream ones (at the top of the column) for 0.2 0.7 Pa. However, a total gas pressure difference is about $1.85 \mathrm{~Pa}$ for static equilibrium of gas between the ends of the vertical soil column. This pressure difference may create an upward advection to overcome part of the downward movement of the TCE vapor due to gravitational force. The measured upstream and downstream vapor concentrations kept increased until steady state reached at about 5 and 10 hours, respectively, after the experiment started. The upstream vapor concentration reached about one tenth of saturated vapor concentration of TCE (about $0.03 \mathrm{~g} / \mathrm{L}$ ) in the experiment and is a little bit greater than the downstream one for about $0.001 \mathrm{~g} / \mathrm{L}$ at steady state. After comparing the model simulations with the experimental data and studying the sensitivities of several parameters on TCE density evolutions from a source, two conclusions are drawn:

1. For a source with TCE vapor concentration of $0.03 \mathrm{~g} / \mathrm{L}$ (the experimental condition), both models predict similar downstream vapor density evolution profiles which match the measurements well. Downward density-driven flow is counter balanced by upward advection due to total pressure gradient arisen from static equilibrium of gas for this source condition.

2. For a source with TCE vapor concentration of about $0.5 \mathrm{~g} / \mathrm{L}$ (saturated TCE vapor concentration), the TCE density evolution profiles predicted with MISER are substantially over the DGPT results at the downstream locations. Because of different diffusive approaches and formulations employed in the two models, the over-prediction of MISER is more substantially for high source density conditions as compared to the DGPT results.

\section{References}

[1] L. M. Abriola, J. Lang and K. Rathfelder. Michigan soil vapor extraction remediation (MISER) model: a computer program to model soil vapor extraction and bioventing of organic chemicals in unsaturated geological material. 
U.S. Environmental Protection Agency, Office of Research and Development, National Risk Management Research Laboratory, Cincinnati, OH, 1997.

[2] R. W. Falta, I. Javandel, K. Pruess, P. A. Witherspoon, "Density-driven flow of gas in the unsaturated zone due to the evaporation of volatile organic compounds," Water Resources Research, vol. 25, no. 10, pp. 2159-2169, 1989.

[3] C.-S. Fen, L. M. Abriola, "A comparison of mathematical model formulations for organic vapor transport in porous media," Advances in Water Resources, vol. 27, no. 10, pp. 1005-1016, 2004.

[4] C.-S. Fen, "Assessing Vadose Zone Biodegradation by a Multicomponent Gas Transport Model," Vadose Zone Journal, vol. 13, no. 1, 2014. DOI:10.2136/vzj2012.0179.

[5] C.-S. Fen, Y. Sun, Y. Cheng, Y. Chen, W. Yang, C. Pan, "Density-driven transport of gas phase chemicals in unsaturated soils," Journal of Contaminant Hydrology, 2017. https://doi.org/10.1016/j.jconhyd.2017.12.003 